\title{
Allergen-induced airway remodelling
}

\author{
C.M. Lloyd* and D.S. Robinson**\#
}

\begin{abstract}
Airway remodelling is associated with chronic asthma but it remains unclear whether it results from airway inflammation in response to allergens or immune-mediated events such as viral infections. Although the acute inflammation associated with asthma has been modelled extensively both in vitro and in vivo, the structural changes occurring in the lung have only recently been investigated. These in vitro, in vivo and in silico systems have been designed to examine the pathways leading to allergen-induced airway remodelling and have enabled investigators to draw conclusions about the participation of key cells and molecules in the development of allergen-induced airway remodelling. However, fundamental questions remain regarding the genesis of remodelling as well as the relationship between functional symptoms and pathological changes that occur. In this review the key questions relating allergen exposure to development of remodelling are discussed, as well as the steps that are being undertaken to investigate them.
\end{abstract}

KEYWORDS: Airway function, airway remodelling, asthma, eosinophils, inflammation
$\mathbf{A}$ irway remodelling is a feature of asthma. It is a collective term that can be defined as the presence of persistent changes to normal airway structure involving changes in the composition, organisation and function of structural cells, as well as enhanced turnover of extracellular matrix components (fig. 1). Structural changes include subepithelial fibrosis, which contributes to the thickening of airway walls due to the deposition of extracellular matrix proteins such as collagens, laminin and tenascin. Increased myocyte smooth muscle mass, also indicative of airway remodelling, is thought to be due to an increase in myofibroblast proliferation or recruitment, and further enhances extracellular matrix deposition. Mucous gland hyperplasia leads to excessive mucus secretion and can, in severe cases, lead to occlusion of the airways. Increased bronchial vascularity has also been documented. Thus, airway remodelling results in thickened airway walls in asthma. The physiological consequences of these changes remain uncertain, in part because these changes are not fully reversed by current asthma therapy. However, airway remodelling is postulated to be a determinant of airway hyperresponsiveness
(AHR) as well as the accelerated loss of lung function over time that is documented in many asthmatics.

This collective description of airway remodelling was originally generated on the basis of descriptive pathological studies of biopsies either taken post mortem from patients who died from asthma, or more recently by fibreoptic bronchoscopy in volunteers with asthma [1]. Such studies have demonstrated changes of remodelling even in mild asthma and in children, and most studies have failed to relate asthma severity to the degree of airway remodelling. While these observational studies are important and have yielded important information regarding tissue structural changes, they cannot impart much about the natural history of remodelling or determine potential therapeutic targets. Important questions that remain unanswered include the following. How is airway remodelling initiated? How is it related to airway inflammation or how does it represent a separate genetic determinant of asthma? Are the changes reversible? How important is it as a therapeutic target? Interventional studies were hampered by the lack of laboratory models but a variety of modelling approaches have now been described
Previous articles in this Series: No. 1: Fixman ED, Stewart A, Martin JG. Basic mechanisms of development of airway structural changes in asthma. Eur Respir J 2007; 29: 379-389. No. 2: Bergeron C, Tulic MK, Hamid Q. Tools used to measure airway remodelling in research. Eur Respir J 2007; 29 : 596-604.
AFFILIATIONS

*Leukocyte Biology Section, and ${ }^{\#}$ Allergy and Clinical Immunology, National Heart and Lung Institute, Faculty of Medicine, Imperial College London, London, UK.

\section{CORRESPONDENCE}

C.M. Lloyd

Leukocyte Biology Section

National Heart and Lung Institute

Faculty of Medicine

Imperial College London

London

SW7 2AZ

UK

Fax: 442075943119

E-mail: c.lloyd@imperial.ac.uk

Received:

December 212005

Accepted after revision:

October 102006

SUPPORT STATEMENT

C.M. Lloyd and D.S. Robinson were

both supported by the Wellcome

Trust UK.

STATEMENT OF INTEREST

None declared.

European Respiratory Journal

Print ISSN 0903-1936

Online ISSN 1399-3003 


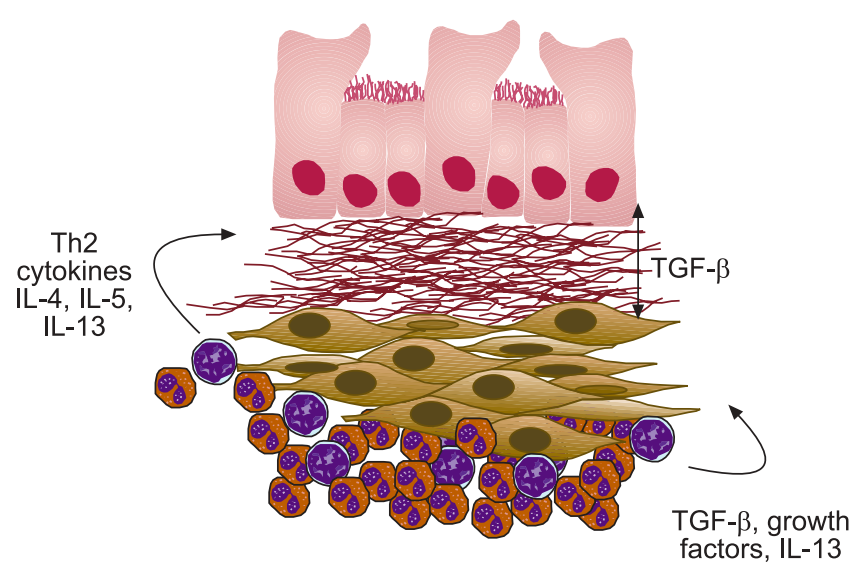

FIGURE 1. Mediators involved in airway remodelling. Th2: T-helper cell type 2; IL: interleukin; TGF: transforming growth factor

to address mechanistic questions and allow pre-clinical intervention studies.

\section{MODELS TO STUDY AIRWAY REMODELLING In vitro cell cultures}

Classical in vitro cell-culture systems have been enormously valuable in delineating some of the functions that lung structural cells might play in the development of airway remodelling. These systems have not specifically examined allergen-induced events but rely on the addition of mediators such as T-helper cell (Th) type 2 cytokines to the cells in culture. Cultures of epithelial cells, fibroblasts and smooth muscle cells using immortalised cell lines established that these cells were capable of more than merely maintaining the structural integrity of the lung. Experiments have determined that these cells are able to respond to, and in many cases secrete, a range of inflammatory mediators, including cytokines and chemokines, as well as profibrotic factors such as transforming growth factor (TGF)- $\beta$. However, in order to try and obtain information more relevant to the lung, primary cells have also been isolated from the lung. Normal lung fibroblasts, epithelial cells, smooth muscle cells and vascular endothelial cells can be isolated from surgical specimens or post mortem, and are now available commercially. In addition, it is possible to obtain these cells from allergic as well as nonallergic patients [2], enabling comparisons to be made. In vitro cultures of these cells have enabled researchers to examine the effects of inflammatory or profibrotic mediators on a wide range of functions, including proliferation, mediator secretion, extracellular matrix metabolism, protease secretion and, in some cases, migration. The culmination of these experiments is the recognition that lung resident cells are integral to the development and maintenance of lung inflammation and remodelling.

\section{Epithelial cells}

Primary lung epithelial cells have been isolated from cadavers and are available commercially. When grown on plastic in submerged culture, epithelial cells have been shown to secrete a range of inflammatory mediators and have the capacity to present antigen. Therefore, it has been postulated that epithelial cells make an active contribution to the generation of allergic airway inflammation after allergen challenge. However, investigators have more recently developed more sophisticated air-liquid interphase culture (ALI) techniques to allow their differentiation into mucus and ciliated cells. The apical secretions from normal human tracheobronchial epithelial cells cultured in this system were found to contain mucinlike materials as well as lysozyme, lactoferrin and secretory leukocyte protease inhibitor [3]. Others have also shown that normal human bronchial epithelial cells grown on microporous inserts at ALI express a well-differentiated mucociliary phenotype compared with cells grown on plastic in submerged cultures, which tend to be poorly differentiated. Furthermore, responses to the Th2-type cytokines interleukin (IL)-4 and IL-13 are highly dependent on the culture technique; cells cultured on plastic exhibited significant concentration-dependent increases in granulocyte-macrophage colony-stimulating factor and TGF$\beta_{2}$ secretion, whereas cells grown at ALI showed no statistically significant response [4]. These results suggest that functions such as cytokine secretion may be critically dependent on the cell-culture technique employed and that results from in vitro cultures must be interpreted with caution.

Increasing evidence also shows that epithelial cells play a crucial role in the development of airway remodelling and it has been further suggested that these may act independently of inflammatory events. Epithelial shedding and hypertrophy, particularly of the goblet cells, are indicative of remodelled airways and many investigators have used isolated epithelial cells to try and determine how they contribute to airway fibrosis. One emerging theory regarding the contribution of epithelium proposes that an inability to appropriately repair damage to the epithelium leads to activation of the attenuated myofibroblast sheath (part of the embryological epithelialmesenchymal trophic unit (EMTU)), leading to the deposition of further myofibroblasts and extracellular matrix (ECM) proteoglycans. Evidence in support of this comes from the fact that asthmatic epithelium has an increased susceptibility to oxidant injury and an inability to repair rapidly. Asthmatic epithelium has a high expression of p21 ${ }^{\text {waf }}$, an inhibitor of cell cycle progression [5]. This regulator of G1 cyclins can be induced by TGF- $\beta$. Epidermal growth factor receptor is also upregulated in the epithelium of patients with chronic asthma, although paradoxically there is no evidence for increased proliferation of the epithelium [6]. It has been suggested that in asthma there is reactivation of the EMTU, which is involved in the development of airway structure during embryological development, thus contributing to the long-term changes seen in the remodelled asthmatic airway. These findings have led to the suggestion that the bronchial epithelium is fundamental to the development of allergic pathology, and that the relationship between the bronchial epithelium, stem cells and the mesenchyme is crucial. It is proposed that epithelial damage and Th2 cytokines cooperate to promote functional disturbance of the EMTU, leading to myofibroblast activation and induction of the inflammation and remodelling that is characteristic of chronic asthma [7]. It is also suggested that this might result from inherited susceptibility genes such as disintegrin and metalloproteinase (ADAM)33 [8], which is a matrix-active enzyme. These theories, however, have not been fully investigated. The ideas stem from in vitro investigations with isolated epithelial cells, but interactions between the different 
cell populations making up the EMTU have not been studied either in vitro or in vivo. The future challenge will be to design systems which enable these hypotheses to be tested. One study has used laser capture microdissection to separate the bronchial wall epithelium and smooth muscle in lung sections from mice chronically exposed to allergen. Gene expression of TGF- $\beta_{1}$ and plasminogen activating inhibitor- 1 were determined to be significantly upregulated only in the airway epithelium, implicating these cells in the pathogenesis of airway remodelling [9].

\section{Smooth muscle cells}

Smooth muscle cells are increasingly regarded as important in the development of lung pathology during asthma. In vitro culture of these cells has shown that they are able to produce cytokines and chemokines that promote the recruitment of inflammatory cells and lead to the activation of other lungresident cells that participate in the development of the remodelled airway. Infiltration of the airway smooth muscle by mast cells has been shown to be the main distinguishing feature between asthma and eosinophilic bronchitis (a condition incorporating cough with eosinophilic airway inflammation in the absence of AHR), and the interaction of these cell types is the subject of intense investigation [10, 11]. Furthermore, increased smooth muscle mass is postulated to contribute to the development of AHR [12]; therefore, understanding the signals that lead to increased proliferation or recruitment of smooth muscle is important.

Another possible contributor to smooth muscle mass is the presence of myofibroblasts. These are a specialised type of fibroblast that constitutes an important component of fibrotic reactions in many tissues and diseases. In asthma, myofibroblasts are increased in the lamina reticularis and lie in close proximity to the smooth muscle bundles of the airway. The in vivo origins of myofibroblasts are not well understood but they are thought to derive from either smooth muscle or from fibroblasts. Recent studies have determined that progenitor cells termed fibrocytes are recruited to areas of tissue injury and then differentiate to form fibroblasts [13]. These fibrocytes are circulating cells distinguished by their unique characteristic of expressing the haematopoietic stem cell antigen CD34 in conjunction with collagen I, and are thought to contribute to wound healing and fibrotic reactions in vivo. Once recruited to the site of tissue injury or inflammation, fibrocytes are thought to differentiate into myofibroblasts, gaining expression of a smooth muscle actin but losing expression of CD34. Allergen exposure has been shown to induce the accumulation of these fibrocytes in the bronchial mucosa of patients with allergic asthma [14]. These results indicate that circulating fibrocytes might function as precursors of myofibroblasts and thus contribute to the pathogenesis of airway remodelling in asthma, but this has not yet been demonstrated directly.

\section{Mixed cell cultures}

The vast majority of in vitro studies have been carried out on isolated cells cultured in suspension with recombinant proteins. It is questionable whether these cells provide much information about the potential function of the particular cell type in vivo. However, there are a number of models that are being developed that place the cells on a support to allow three-dimensional culture or interaction of cells to model the role of matrix proteins in the three-dimensional milieu of the airways in asthma. These models aim to retain the advantages of isolated tissue assays but also allow continuous investigation of long-term events. These models are needed to facilitate the study of changes in pharmacological properties, of intraand intercellular processes that characterise AHR. One study attempted to "engineer" bronchial mucosa by incorporating human bronchial fibroblasts from either normal or asthmatic donors into collagen gel and then seeding bronchial epithelial cells over this gel before culture in an ALI in the presence or the absence of T-lymphocytes [15]. Histological analysis showed that engineered mucosa with normal bronchial cells exhibited a pseudo-stratified ciliated epithelium with the presence of mucus secretory cells. These features were comparable with those observed in normal bronchial tissues. In contrast, engineered mucosa from asthmatic subjects showed a disorganised tissue structure, particularly with respect to the epithelial cell arrangement. The percentage of IL-5-positive T-cells was significantly higher in engineered bronchial mucosa from asthmatic subjects compared with mucosa from normal volunteers.

Cultures of bronchial biopsy explants have also been developed to try and take into account interactions between different populations of cells. One such bronchial explant system sought to gain insight into which cytokines are involved in perpetuating allergen-induced inflammatory processes in the asthmatic airways [16]. The results showed that bronchial biopsy tissue maintained in culture for $24 \mathrm{~h}$ actively transcribes cytokine mRNA without any overt stimulation. It was possible to stimulate the explants with allergen in vitro and monitor cytokine responses. Clear differences in the spectrum of cytokine expression between atopic asthmatic and normal control tissue were observed. Stimulation with Der $\mathrm{p}$ allergen did not alter the cytokine profile of biopsies from control individuals, but elevated the expression of IL-5 and -13 mRNA and significantly induced the secretion of the protein by the asthmatic airway tissue. Another three-dimensional organ culture system used portions of guinea pig airways and showed that airways became hyperresponsive to a range of different agonists while maintaining their three-dimensional organisation [17]. These models have potential for testing future therapeutic targets.

\section{In vivo models}

Animal models

Mouse models of allergic inflammation have been useful in defining roles for particular inflammatory mediators or cells. Historically, these have generally involved systemic sensitisation with a protein in conjunction with an adjuvant, followed some weeks later by local inhalation challenge via the airway with the same protein allergen [18]. Even with the variety of results obtained due to using different protocols and strains of mice, these models have yielded a substantial amount of information regarding pathophysiological processes after allergen challenge in vivo, and the use of knockouts, transgenics and administration of antibodies or pharmacological inhibitors has led to the identification of multiple pre-clinical targets. More recently, investigators have developed longerterm challenge protocols (often involving several months of 
challenge) in order to try and replicate the more chronic aspects of asthma seen in patients. Generally these "chronic" models demonstrate considerably less eosinophilic inflammation in the lungs, but lungs show evidence of remodelling with increases in collagen deposition, smooth muscle mass and goblet cell hyperplasia [19, 20]. Interestingly, continued use of an environmentally relevant allergen, house dust mite, has been shown to elicit a chronic inflammatory response together with structural remodelling, even in naïve, unsensitised mice [21]. Consequently, studies are now emerging in which the relationship of individual cell types and specific mediators with the development of remodelling events is being investigated. IL-13 is thought to be a critical mediator in airway remodelling since IL-13 knockout mice, or treatment with neutralising antibodies, ameliorates many of the symptoms associated with chronic allergen challenge [22-24]. Lack of IL-5 is also beneficial in reducing airway remodelling [25], perhaps due to effects on eosinophils, which play an important role in its development [26].

Transgenic technology has also been used to develop model systems to study airway remodelling in vivo. Interestingly, lung-specific expression of many of the Th2-type cytokines results in the development of airway remodelling spontaneously, without the need for any allergen challenge. In particular, transgenic expression of either IL-13 or -9 driven by a lung-specific promoter results in excessive mucus secretion and increased ECM deposition in the airways [27, 28]. However, it could be argued that, particularly for IL-13, the observed pathology is more comparable to that seen in chronic obstructive pulmonary disease rather than chronic asthma. In addition, although these transgenic models allow observation of the consequences of high levels of a particular mediator, they may overemphasise the contribution of that particular molecule; it is probable that the inflammatory mediators associated with asthma function as a tightly controlled network to elicit the characteristic pathological changes.

One major criticism of mouse models of airway remodelling has been that the murine airways are relatively simple compared with those of humans. Other models have emerged that use larger animals that are arguably more anatomically similar to humans. A recent sheep model takes advantage of the fact that ovine lung innervation and blood supply is nearer to that of humans than mice [29]. SNIBSON et al. [29] have described a model of airway inflammation and remodelling in sheep induced by chronic inhalation of house dust mite by segmental allergen challenge. These sheep developed an immune response to house dust mite, as characterised by increased allergen-specific immunoglobulin (Ig)E. After a 6month challenge protocol, lung tissue showed evidence of goblet cell hyperplasia, collagen deposition and smooth muscle hyperplasia, but in only three out of the seven sheep studied.

Chronic allergen challenge models have also been established using nonhuman primates, which are arguably closer still in anatomy to humans [30]. Perhaps more importantly, the physiological state of both the respiratory and immune systems are considerably different at birth in mice compared with humans or primates, with development occurring in a matter of weeks in mice and years in humans. Primate models generally involve either challenge with Ascaris in naturally sensitised monkeys or sensitisation and challenge with house dust mite antigen [31, 32]. In the latter model, long-term challenge with house dust mite resulted in eosinophilic inflammation in conjunction with mucus metaplasia. Thickening of the basement membrane was reported, although there was no description of changes in fibrotic mediator production or analysis of ECM components [32,33]. This protocol also induces hypertrophy of the airway smooth muscle, but these changes were not related to AHR or inflammation [34].

\section{Human models}

One of the advantages of studying asthma is that it is possible to induce acute changes in patients by controlled allergen challenge in the clinic. These inhalational challenge protocols have been used for many years to study the mechanisms of asthma in affected individuals. Sensitised atopic asthmatics show an early asthmatic response within 15 min of segmental allergen challenge that is characterised by airway narrowing measured by reduced forced expiratory volume in one second (FEV1). This phenomenon is thought to occur largely as a result of mast cell degranulation after IgE binding. Around half of these individuals will then experience a later fall in FEV1 $\sim 3-8 \mathrm{~h}$ after challenge, with an accompanying increase in AHR which might persist for days or weeks [35]. This late asthmatic reaction has been the subject of much investigation. It is characterised by an influx of eosinophils, Th2 cells and basophils and has been used as a model of airway inflammation in many drug-development programmes. Interestingly, it has been more recently demonstrated that this protocol also leads to activation of the EMTU and upregulation of several molecules and mediators that are indicative of the remodelling process. PHIPPS et al. [36] examined endobronchial biopsies obtained before and $24 \mathrm{~h}$ after inhalational challenge of mild asthmatics for evidence of ECM deposition in the reticular basement membrane (RBM). Allergen challenge was shown to increase the number, of fibroblasts that were synthesising collagen as well as the thickness of tenascin in the RBM. Increases in the number of epithelial cells positive for the TGF$\beta$ signalling molecule, phospho-Smad2, were also seen after allergen challenge. Thus, allergen challenge in patients with mild asthma induces activation of epithelial cells and fibroblasts in the EMTU, as well as increasing production of ECM proteins within the RBM. The authors concluded that airway remodelling in asthma may, in part, result from repeated acute activation of the EMTU by allergen exposure [36]. How remodelling is activated in nonatopic asthma and the role of viral infections (persistent or transient) remain important unanswered questions.

\section{MODELLING REMODELLING}

An emerging area of research aims to negate the use of animal or human models. Mathematical modelling using basic fluid dynamics to model airway resistance has been applied to examine the effects of airway smooth muscle shortening and airway wall thickening on changes in pulmonary resistance [37]. Studies using this technique have determined that increased muscle mass is likely to be the most important abnormality responsible for the increased resistance observed in asthma [38]. Others have used similar systems in order to 


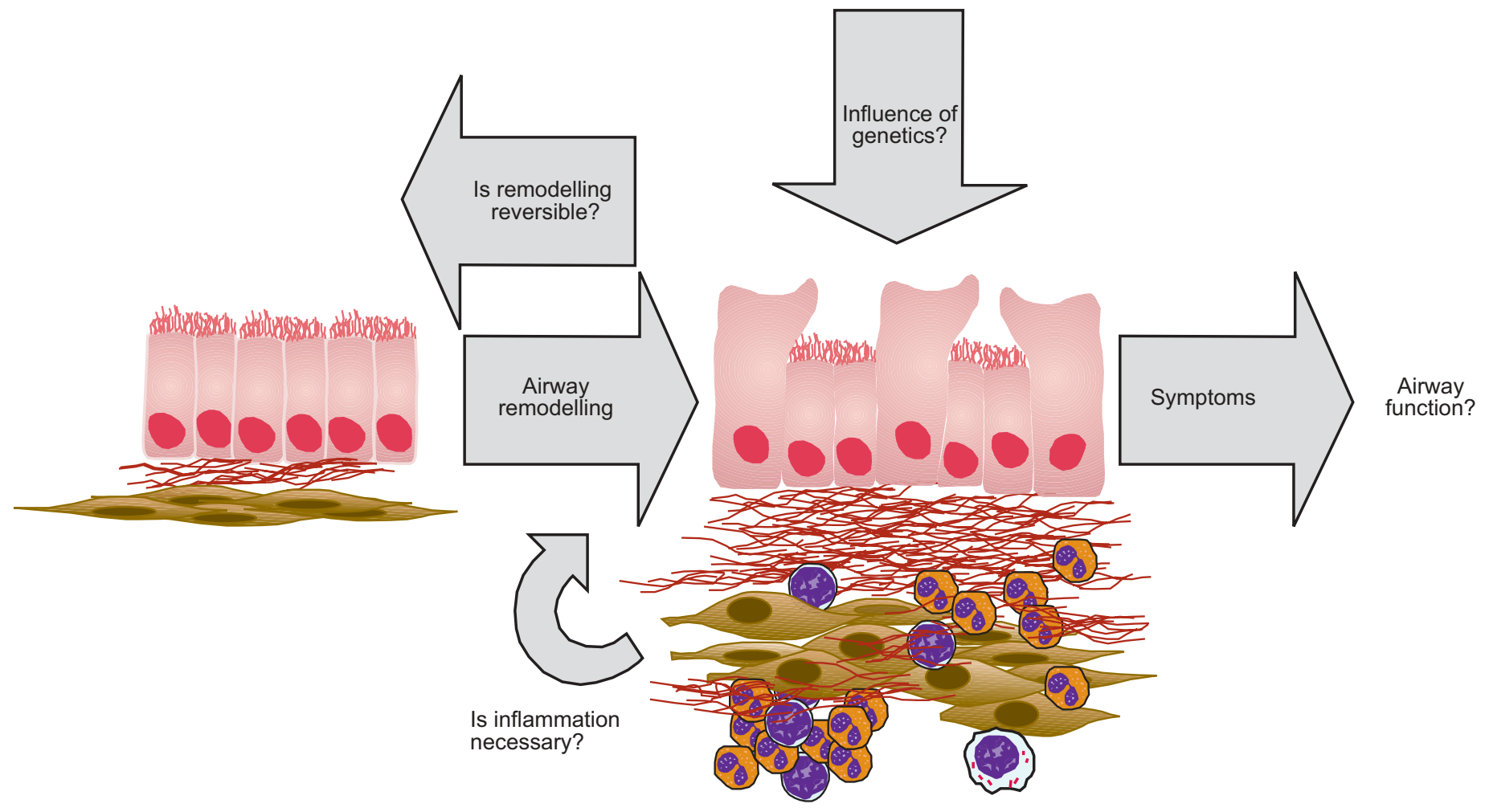

FIGURE 2. Critical questions in airway remodelling today.

predict the consequences of phenomena, such as airway narrowing [39] or changes to the integrity of the mucosa [40].

Computer or in silico modelling of the airways have also been investigated in order to make correlations between structure and function. Structural models of the airways have been generated to take into account airway dimensions and connectivity, and have then been applied to the study of lung mechanics and gas exchange. Until recently these models did not take into account spatial arrangement of the airway structure. However, the rapid advancement of three-dimensional imaging of the lung has strengthened clinical diagnosis of various lung diseases and has improved the development of three-dimensional lung models to include vascular branching [41]. Structure-function relationships of the tracheobronchial tree have been studied by three-dimensional modelling of airway-parenchymal interactions to try to generate simulation models of the airways during disease. Computer-generated three-dimensional displays of human lungs based on raw data from magnetic resonance imaging have been described. Transverse slices of lung were stacked, compiled and used as a source to generate images of control and asthmatic lungs [42]. In generating a model of the asthmatic lung the following features of asthma were considered: bronchoconstriction of the smooth muscle; inflammation of the airway wall; thickening of the mucus layer; and that the combination of these three effects results in a reduction in the diameter of the airway lumen. The authors point out, however, that the relative magnitudes of these components may be anticipated to vary between different human subjects. Importantly, the authors did not consider the effects of increased matrix deposition, i.e. the effects of remodelling have not yet been simulated by computer. However, the mathematical descriptions of the changes in human lungs produced by asthma have been developed and reflect the heterogeneity and severity of asthma. The physical manifestation of asthma was formulated in the large and central airways and the small airways of the tracheobronchial compartment. Severity of asthma was simulated by reducing airway diameters by $20-40 \%$ due to bronchoconstriction, inflammation and mucus. The resultant "asthma model" was used to calculate the effects of disease on the deposition of inhaled particles. For the diverse conditions simulated (e.g. particle sizes, ventilatory parameters, disease locations, asthma severities) airway congestions consistently produced notable increases in particle deposition at the affected site [42]. In the future similar in silico models could be developed in an attempt to gain more information regarding the relationship between airway structure and function.

\section{TODAY'S QUESTIONS}

There has been a significant increase in the interest and amount of investigation into the mechanisms behind airway remodelling in patients and the development of more relevant in vivo and in vitro models to study these mechanisms. However, there remain several critical questions (summarised in figure 2), as follows. 1) What is the relationship between airway function and airway remodelling: is airway remodelling the cause of asthma symptoms and morbidity or merely a byproduct of airway inflammation? 2) Is inflammation necessary for the development of airway remodelling; are they parallel or sequential events? 3) Is airway remodelling reversible? 4) Is airway remodelling genetically determined? 

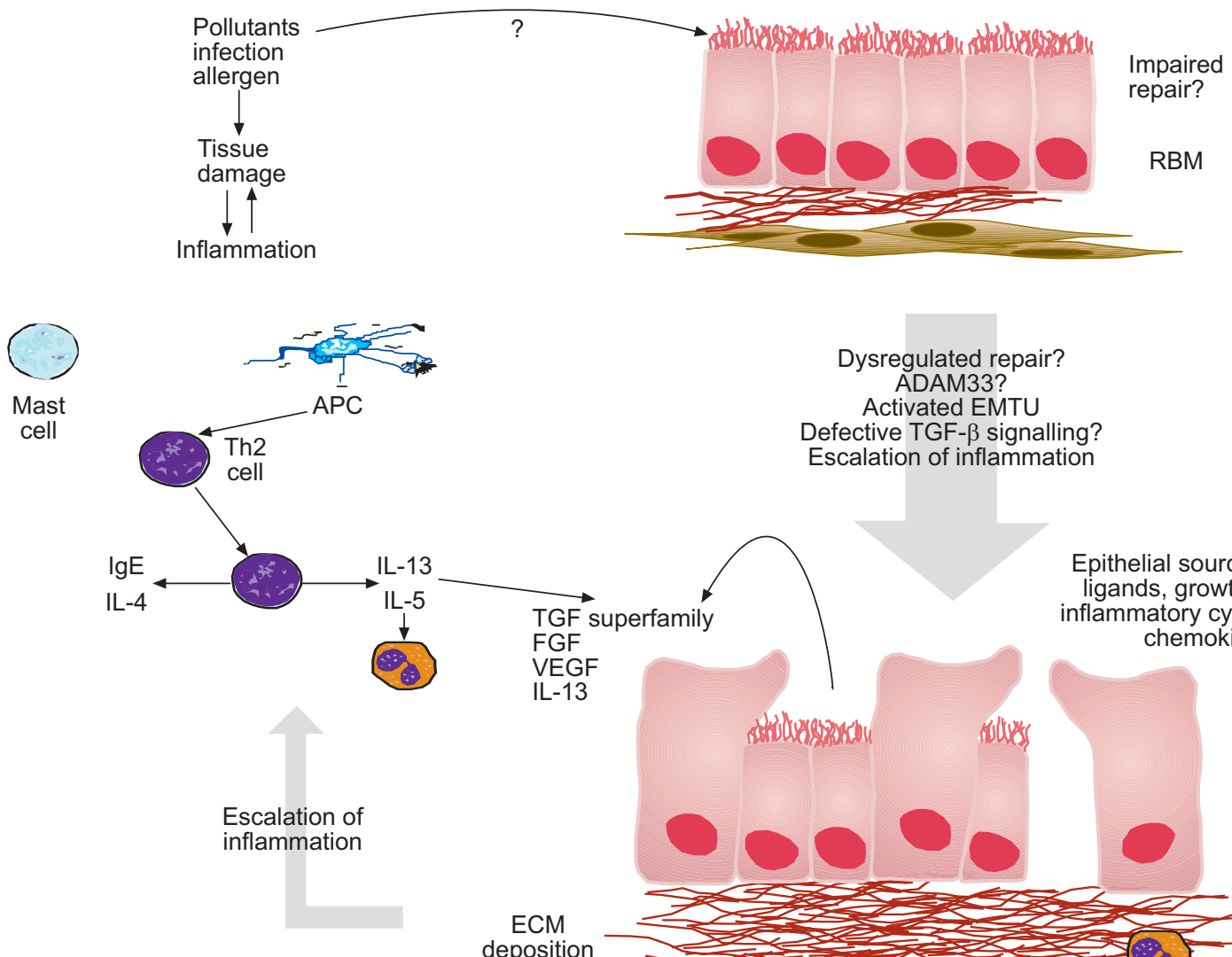

ASM hypertrophy-hyperplasia Vascular remodelling-oedema ASM myositis?
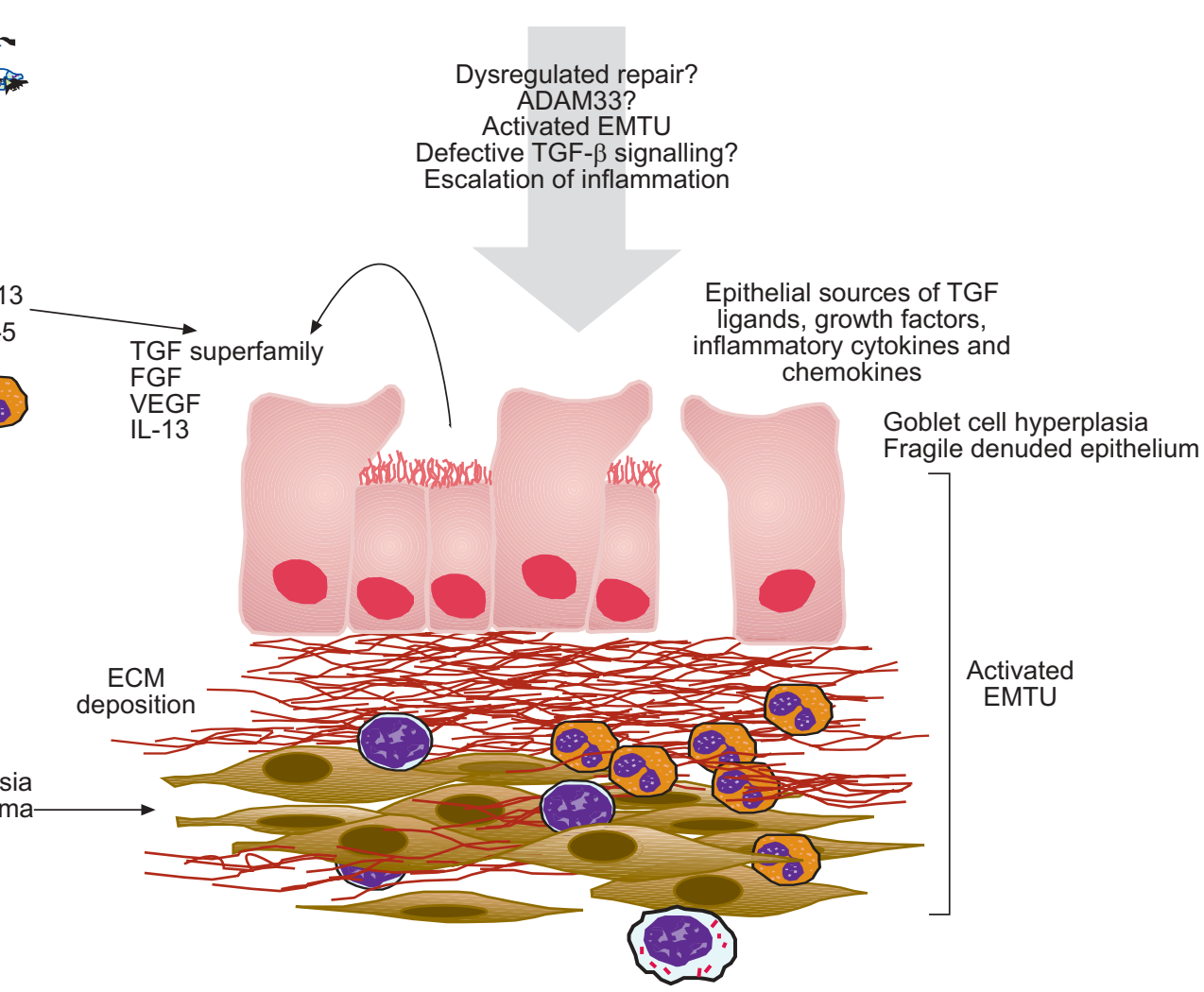

AHR

Airway obstruction

FIGURE 3. Summary of current concepts in the pathogenesis of airway remodelling in allergen-induced asthma. Exposure to environmental insults can lead to airway damage, and individuals with a genetic predisposition to atopy and/or dysregulated or impaired airway repair may go on to develop sustained inflammation and tissue remodelling. Increased activation of the epithelium leads to signalling to inflammatory cells and also activation of the underlying mesenchymal cells (activated epithelialmesenchymal trophic unit (EMTU)). Both structural and inflammatory cell sources of growth factors, such as those of the transforming growth factor (TGF)-superfamily as well as vascular endothelial growth factor (VEGF) and interleukin (IL)-13, are thought to be important. Progressive structural changes include increased numbers and size of fibroblasts and airway smooth muscle and vascular remodelling together with excessive and dysregulated extracellular matrix (ECM) deposition, the balance of which may lead to a phenotype that is characterised by increases in airway hyperresponsiveness (AHR) or airway obstruction. In turn the pro-inflammatory environment generated by chronic structural cell activation will sustain and propagate the inflammatory response to ongoing environmental insults. RBM: reticular basement membrane; APC: antigenpresenting cell; ADAM: disintegrin and metalloproteinase; Th2: T-helper 2; Ig: immunoglobulin; FGF: fibroblast growth factor; ASM: airway smooth muscle.

\section{What is the relationship between airway function and airway remodelling?}

Perhaps one of the most urgent questions relating to the development of airway remodelling is that of the functional consequences of the remodelling for the patient. The attempts to make correlations between the development of tissue structural changes and changes in lung function parameters have been controversial and remain the subject of intense discussion and investigation. Changes in airway function include hypersensitivity (a leftward shift in curves for bronchoconstrictor dose-response), hyperreactivity (increased slope of these curves) and a greater maximum degree of induced bronchoconstriction. Longitudinal studies, however, imply that AHR should be considered as an independent risk factor for development of asthma rather than as an outcome itself [43]. Multiple studies have determined that changes in airway function are accompanied by inflammatory infiltrates composed of eosinophils, T-cells, monocytes and neutrophils and cytokines characteristic of a classical Th2 response. This implies that AHR is at least partially dependent on inflammatory events [44-46], but the exact contribution of inflammation to airway dysfunction remains ill-defined [47]. The observation that AHR persists in patients despite prolonged treatment with anti-inflammatory corticosteroids [48-50] implies that other 
mechanisms account for a major component of airway dysfunction.

The clinical consequences of airway remodelling remain uncertain but contributions to AHR and fixed airflow obstruction have been suggested. Asthmatic subjects show an accelerated decline in lung function compared with nonasthmatics, and this is more marked in those asthmatics that smoke $[51,52]$. Research has been hampered by the fact that there are no noninvasive methods of assessing airway remodelling at present, and human airway research is limited to mainly morphological and in vitro experimental work that is constrained further by ethical limitations. Nevertheless, recent bronchoscopic studies have enabled hypotheses to be formed regarding the role of particular mediators and cells in the development of structural changes following allergen challenge [36, 43, 53]. In addition, high-resolution computed tomography (HRCT) shows promise in evaluating structurefunction relationships in asthma [54]. HRCT reveals that an increase in the ratio of bronchial wall thickness to bronchial diameter exists in the large airways in asthma [55]. Interestingly, wall thickness as assessed by HRCT correlates with subepithelial fibrosis, as assessed by biopsy [56]. In addition, HCRT wall thickness is associated with increased airflow limitation and correlates with asthma duration, as well as traditional indices of asthma severity and control [43]. In these computational models the increase in reactivity and maximal response caused by airway thickening is related to one or a combination of mechanisms. Increase in the airway wall thickness internal to the smooth muscle layer can amplify the airway narrowing for a given degree of airway smooth muscle shortening. Increase in the thickness of the adventitial layer has the potential to uncouple the airway smooth muscle layer from the surrounding parenchyma, allowing the smooth muscle to shorten more before being balanced by parenchymal tethering. Increase in the smooth muscle layer could result in greater force development and therefore shortening against the loads that normally attenuate airway muscle shortening [57]. Paradoxically, a recent study determined that airway reactivity negatively correlated with airway wall thickness. Airway sensitivity was related to the degree of airway inflammation as determined by sputum eosinophilia, but not to any index of airway wall thickness. It was concluded that airway wall thickening was perhaps protective against excessive airway narrowing in asthma [58]. This apparent contradiction of accepted dogma might be explained by the fact that the computational studies undertaken are primarily based on altered geometry and do not fully take into account the potential effect of airway wall thickening on the mechanical properties of the airway. It has been argued that there are several potential explanations to this apparent paradox, all relating to the stiffness of the airways. Deposition of ECM could separate the smooth muscle layer from the parenchyma, thus limiting the ability of the smooth muscle to narrow the airway lumen. Similarly, thickening of the airway wall between the smooth muscle layer and the epithelium might stiffen the airway. In addition, although the smooth muscle layer is increased during chronic asthma, it has been argued that it changes from a mainly contractile phenotype to a secretory phenotype [59]. Therefore, the apparent paradoxical relationship between airway geometry and AHR described by
NiIMI et al. [58] may simply be a function of the mechanical properties of the material deposited within the layers of the airway wall. However, these results suggest that the present author's ideas regarding the relationship between airway remodelling and airway function are simplistic; the mechanical properties of the material thickening the airway wall influence the ultimate change in physiological response, not just the amount [57]. It is clear that some of the changes characteristic of airway remodelling occur in other chronic lung diseases and are not specific to asthma.

Investigating the relationship between airway remodelling and changes in airway function in humans is difficult, since endobronchial biopsies do not always show the true extent of changes to the airway wall and indeed serial biopsies from the same patient are largely impractical and unethical. At present, there is a lack of reliable noninvasive predictive markers and insufficient data from longitudinal studies to define the natural history of remodelling from childhood wheezing into adulthood. The development of such noninvasive systems is urgently needed in order to further the analysis of the relationship between airway remodelling and changes in lung function. In the absence of suitable human studies, animal models can attempt to dissect the relationship between lung structural changes and lung function after allergen challenge. The majority of mouse models report some change in lung function after prolonged challenge [25, 60-63], which correlates with airway inflammation and airway remodelling. However, the relationship between airway inflammation, airway remodelling and changes in airway function is still ambiguous. Several of these studies argue that airway structural changes can be uncoupled from AHR. One elegant study compared brief or chronic exposure of mice to allergen and determined that airway dysfunction and remodelling persisted beyond the resolution of immune-mediated inflammatory events [62]. Airway responses to methacholine were measured and increases in the maximal inducible bronchoconstriction persisted for $\geqslant 8$ weeks after cessation of allergen challenge. These functional changes were seen in conjunction with increases in contractile tissue in the airway wall. In contrast, observed increases in airway reactivity (rate of increase in respiratory resistance for a given dose of methacholine) only persisted beyond resolution of allergen-induced inflammation in chronically challenged mice. The authors concluded that sustained airway hyperreactivity was not associated with ongoing Th2 inflammatory markers, such as eosinophilia or IL-13, but that sustained dysfunction occurred as a consequence of airway remodelling rather than these immune-mediated events. One can speculate that the initial development of airway dysfunction is dependent on acute inflammatory responses to allergen challenge, such as recruitment of eosinophils and Th2 cells and production of Th2 cytokines, but that sustained dysfunction is dependent on structural changes to the airways, such as changes in the smooth muscle layer and increased deposition of ECM. Subsequent studies using knockout mice revealed that IL-4 and -13, but not IL-5, are critical for the development of sustained airway remodelling and AHR [64]. However, it could be argued that since these mice are completely devoid of the individual cytokines for the duration of the entire protocol, the observed effect may reflect differences in the initial development of remodelling or AHR rather than in the 
persistence. Studies with neutralising antibodies during different phases of the protocol would determine this.

Intervention studies in mouse models of chronic allergen challenge have revealed some interesting correlations between airway inflammation, development of airway dysfunction and airway remodelling. Several of these models have shown that it is possible to uncouple airway function from either inflammation or airway remodelling. Mice genetically deficient in eosinophils are protected from development of remodelling but, surprisingly, not from changes in airway function [26]. These mice develop robust AHR to acute and chronic allergen challenge but fail to develop airway fibrosis or airway smooth muscle hypertrophy after chronic challenge. Another study has shown that airway inflammation and AHR is preserved in mice that lack mast cells, but that allergen-induced subepithelial fibrosis was partially attenuated [65]. Therapeutic administration of anti-TGF- $\beta$ antibody after the onset of eosinophilic inflammation and AHR had no effect on these parameters but did prevent the development of airway remodelling [66]. These studies show that it is possible to affect one parameter of chronic allergen challenge without affecting others, and will permit the testing of potential therapeutic treatment regimens on outcomes of the various aspects of chronic allergen challenge. In the future it will be important to consider the success of these therapeutic regimes on facets of chronic allergen challenge, such as airway remodelling rather than just eosinophil recruitment. In addition, it will be important to test potential therapeutic treatments using therapeutic rather than prophylactic regimens.

These developments in animal models will increasingly allow dissections of potential components contributing to airway remodelling and their impact in airway physiology. However, relating these findings to chronic asthma in humans remains a challenge. The use of monoclonal antibody therapy in asthma and allergen challenge models may give some insights. For example, FLOOD-PAGE et al. [53] reported reduced subepithelial tenascin staining after anti-IL-5 treatment of asthmatics, but this was not associated with any change in AHR, lung function or asthma symptoms. Chronic intervention and observational studies are required but are difficult to both fund and execute. Of importance here is the question of which parameters of lung function relate to asthma outcomes and can these be related to remodelling? For example, tailoring treatment to AHR [49] or sputum eosinophilia [67] reduced asthma exacerbation rates, suggesting that these are important determinants of morbidity, but it will also be important to determine whether remodelling is related to the development of fixed airflow obstruction, the rate of change of airway calibre or the response to acute challenge (allergen or infection).

\section{Is inflammation necessary for the development of airway remodelling?}

Although it is generally held that airway remodelling occurs as a consequence of chronic inflammation induced by repeated allergen challenge, emerging theories challenge this concept. The idea that reactivation of the EMTU is a key feature of induction of airway remodelling has led to the proposal that inflammation and remodelling are parallel rather than sequential events (fig. 3). Communication between the epithelium and the underlying epithelial fibroblast sheet is reminiscent of the processes that drive branching morphogenesis in the foetus, where the epithelium and the mesenchyme function as a trophic unit. Holgate et al. [68] have proposed that the EMTU becomes reactivated during chronic asthma to drive pathological remodelling. This theory may explain the potentially conflicting findings linking inflammatory cells with markers of remodelling, some of which may be consequent upon inflammation while others are not. In addition, the hypothesis might answer some of the controversies regarding inflammation during asthma, such as why the prolonged use of corticosteroids has little or no effect on the natural history of asthma, even if treatment is instigated in early childhood.

The question as to whether remodelling of the airway wall might precede asthma has been investigated by studies of childhood asthma. Data from longitudinal studies indicate that wheezing in early childhood is a marker for subsequent reduced pulmonary function and asthma. The question is whether or not these children have smaller airway diameters that were genetically or pre-natally determined; whether this would predispose them to wheeze in association with insults such as viral infections; and whether atopy subsequently manifests as wheezing and bronchial asthma. Alternatively, pre-clinical bronchial asthma may cause abnormal airway development or remodelling, which leads to reduced airway diameter and then renders pre-school children more prone to wheezing with viral infections as well as after allergen exposure [69]. An early paper compared biopsies from 12-yrold females with bronchial asthma with post mortem samples from lungs taken from similar aged children who died of severe acute asthma. In the living patients there was evidence of airway remodelling, including goblet cell metaplasia and mucus plugging, together with thickening of the bronchial epithelial basement membrane [70]. Furthermore, smooth muscle hypertrophy and inflammatory cell infiltration were also apparent. The children who had died of asthma showed more pronounced peribronchiolar eosinophil infiltration in conjunction with focal loss of the bronchial epithelium. Therefore, it seems that there is evidence of lung structural changes in children; this is reinforced by a retrospective study of bronchial biopsies from children with respiratory symptoms that subsequently developed bronchial asthma [71]. The histological features of asthma, including eosinophilia and thickening of the lamina reticularis underneath the bronchial basement membrane, were present even in pre-school children prior to the diagnosis of asthma. Even though the precise relationship between airway inflammation and airway remodelling is unclear, there is evidence that airway remodelling occurs in early childhood and that in some cases it pre-dates onset of symptoms. Other studies report that loss of lung function is determined mainly in childhood, but these physiological findings have not been correlated with histological evidence of airway remodelling [72, 73]. Perhaps more worryingly, biopsy studies of schoolchildren who remained relatively asymptomatic due to high doses of corticosteroids showed thickening of the lamina reticularis of the basement membrane compared with nonasthmatic children [74]. There is also evidence for early bronchial smooth muscle hypertrophy in biopsies from schoolchildren [75, 76]. In contrast, a recent study has looked for even earlier evidence of remodelling in pre-school children [77]. Examination of the bronchial mucosa 
of infants aged $<2$ yrs demonstrates that the eosinophilic inflammation and RBM thickening that are characteristic of asthma in older children and adults are not present in infants with recurrent wheeze and/or cough or in the presence of reversible airflow obstruction. Moreover, there was no correlation with atopy. Further study is required to determine the relationship between airway inflammation and remodelling in children in order to identify whether early intervention will impact on lung function in later life.

This relationship has not been investigated at all in animal models. All of the studies described thus far have been performed on adult mice and the question of influence of early life events on development of airway remodelling with or without inflammation has not been addressed. Furthermore, the question as to whether remodelling precedes airway inflammation or AHR has also not been addressed, probably due to the lack of suitable models. Current models of allergic inflammation and remodelling, including those described previously, require systemic sensitisation with allergen to induce changes to airway pathology [20]. Transgenic models in which various Th2 cytokines have been ectopically expressed specifically in the lung have determined that airway remodelling does occur without immune sensitisation and allergen challenge, but the remodelling occurred in conjunction with airway inflammation, most obviously eosinophilia [27, 28]. Therefore, it is difficult to determine which came first, the inflammation or the structural changes. Whether similar results would be obtained if the mice were developed in a strain lacking CD4 T-cells remains to be seen. Models which address these specific questions are urgently required, particularly due to the ethical limitations of performing allergen challenge studies or even biopsy studies in children and infants.

\section{Is airway remodelling reversible?}

One of the most critical questions regarding chronic changes to the lung is that of reversibility. It is not clear how effective current asthma therapies are in reducing remodelling. This is critical since it has become obvious that remodelling events occur early in disease development and it is likely that by the time patients present to their physician and are diagnosed they already display markers of airway remodelling processes that are similar to those observed in patients with long-standing disease [78]. Thus, the true extent of reversibility of changes in airway wall remodelling is unclear, as is the relationship between these airway wall changes and ongoing exposure to allergen. Although studies have reported an improvement in parameters of inflammation and AHR in children undergoing allergen avoidance at high altitude [79], airway remodelling was not included in this study, probably for ethical reasons. One study has reported that airway remodelling is reversible after cessation of exposure to isothiocyanates in sensitised individuals [80]. Reversibility is relatively easy to examine in animal models and investigators have approached this by inducing airway inflammation, AHR and remodelling by chronic allergen challenge and then examining the same parameters at different time points after cessation of allergen exposure. LeIGH et al. [62] looked at lungs up to 8 weeks after final allergen exposure and determined that chronic challenge elicited aspects of sustained airway dysfunction and remodelling that persisted beyond the resolution of acute inflammatory events. Similarly, KUMAR et al. [81] observed that persistence of airway wall eosinophilia and mucous cell hyperplasia was dependent on continued allergen exposure, whereas subepithelial fibrosis and epithelial hypertrophy were not. The current authors determined that peribronchiolar fibrosis and airway smooth muscle changes persisted for $\geqslant 4$ weeks following chronic allergen challenge, but inflammation and AHR resolved [82]. A recent report has described a different model in which allergic inflammation and remodelling are elicited after chronic intranasal instillation of house dust mite extract without prior sensitisation [21]. This protocol resulted in classical Th2-type eosinophilic inflammation concomitant with AHR to methacholine and lung structural changes assessed by goblet cell hyperplasia, collagen deposition and peribronchiolar accumulation of contractile tissue. Interestingly, cessation of house dust mite exposure led to resolution of eosinophilic inflammation within 2 weeks but no resolution of remodelling changes and only partial resolution of AHR [21]. The question of reversibility has not been investigated as yet in larger animal models.

These mouse models all allude to the fact that although the inflammatory aspects of allergen challenge are reversible, the lung structural changes are not. However, all of these studies have relied on cessation of allergen challenge to determine reversibility but complete allergen avoidance is not a viable option for many asthmatics. Therefore, it is important to consider whether airway remodelling might be reversible with therapy. Such studies have been undertaken in patients but, unfortunately, the majority of the available evidence regarding regression of airway remodelling in response to classical asthma treatment is contradictory. Reversal of subepithelial fibrosis in allergic asthmatics after prolonged therapy with high-dose corticosteroids has been reported $[49,83,84]$ but this is not a universal finding $[48,85,86]$. Taken together, these studies indicate that airway inflammation, basement membrane thickening and AHR are interrelated and can be improved by corticosteroids but that this occurs over different time scales and with a different dose-response relationship [87]. Unfortunately, it does not seem that corticosteroids are totally protective against long-term airway damage; proof of their effectiveness needs to be collected after further investigation and other therapeutic strategies explored. A recent study with a nonbactericidal dose of a macrolide antibiotic promoted reversal of airflow limitation in chronic lung allograft rejection [88] indicating that, ultimately, it might be possible to reverse airway remodelling.

Again, mouse models have been used to investigate the effect of common asthma therapies on the development of airway remodelling. Corticosteroids have been used at the onset of allergen challenge and fluticasone was shown to be effective in preventing increases in airway laminin but, interestingly, had no effect on AHR [89]. In contrast, the current authors have shown that administration of budesonide is effective in resolving established airway eosinophilia and AHR and prevents development of airway remodelling [90]. Therapy directed at leukotrienes has also shown that airway remodelling can be prevented [91]. However, none of these studies has used a treatment regimen designed to reverse established airway remodelling. In all of the studies, therapeutic agents were administered at the start of allergen challenge $[89,91]$ or 
at a time of established inflammation, but before remodelling was observed [90]. In the future it will be important to use these models to determine the efficacy of standard asthma treatments in reducing established airway remodelling.

\section{Is airway remodelling genetically determined?}

Asthma is a complex inflammatory disorder in which development is thought to be affected by both genetic and environmental considerations. It is commonly held that asthma is caused by multiple interacting genes, some having protective effects and others contributing to disease pathogenesis, but with each gene having its own tendency to work with, and be influenced by, the environment. Therefore, the complex nature of the disease coupled with the substantial locus heterogeneity and environmental influence has made it difficult to uncover the genetic factors that underlie the disorder [92]. Linkage analysis uses polymorphic markers throughout the genome to determine whether certain markers segregate in the affected family members, indicating that they might be located near one or more genes that cause the disease under study. Numerous studies have examined the association between asthma or its related traits and genetic variations in candidate genes [92]. Most of these studies have utilised common clinical manifestations of the disease, such as AHR, elevated IgE and atopy, in order to look for evidence of genetic linkage. However, there is accumulating evidence that airway remodelling is also genetically linked. Genetic mapping studies have revealed that chromosome 5q31-q33 is of particular interest since it contains the cytokine gene cluster including IL-4, $-5,-9$ and -13 , as well as other growth factors and receptors implicated in asthma pathology [93]. It is of interest that transgenic models developed using tissue-specific promoters to drive lung expression of IL-9 and -13 in particular result in airway remodelling as well as the other common features of the allergic syndrome [27, 28]. Linkage disequilibrium studies identified multiple polymorphisms in the ADAM33 gene that are associated with asthma [94]. Haplotypes comprised of polymorphisms within the ADAM33 gene were significantly associated with asthma in the case-control samples, as well as in family based association tests. ADAM33 is a member of the ADAM subgroup of the zinc-dependent metalloproteinase superfamily and is a very plausible candidate gene for tissue remodelling in asthma. The gene has been found to be expressed on fibroblasts and smooth muscle cells. In addition, polymorphic variations in ADAM33 predict impaired early life functions [95] and single nucleotide polymorphisms in ADAM33 have been linked with accelerated decline in lung function in the general population [96]. Although its precise function is not fully understood, it has similarities to other ADAM genes that possess proteolytic activity and this suggests that ADAM 33 may play a role in airway remodelling. The development of a mutant mouse either overexpressing ADAM33 or genetically deficient in ADAM33 is eagerly awaited and might yield some clues as to the role of this molecule in airway remodelling.

The contribution of genes and environment to the development of airway remodelling highlights a major drawback of almost all animal models. Most strains of mice are inbred and are housed in clean environments that may be beneficial when considering the role of a particular pathway, cell or molecule in isolation. However, it is important to consider that innate tissue responses, as well as primary immune responses, are straindependent. Differences in the strain of mouse used for particular experiments have resulted in some contradictory results in the past [97] and are an important factor when comparing similar studies. One study has investigated the effect of mouse strain on the development of airway remodelling by assessing the responses of commonly used strains of mice to airway allergen challenge in the absence of systemic sensitisation [98]. Using this protocol, persistent AHR, eosinophilic inflammation, collagen deposition and airway wall thickening were all found to be strain-dependent. Changes were minimal in the commonly used Balb/c strain and absent in C57BL/6 and $\mathrm{C} 3 \mathrm{H} / \mathrm{HeJ}$ mice. Interestingly, the strain that gave consistent remodelling responses to airway intranasal challenge (rather than inhalational) was the $\mathrm{A} / \mathrm{J}$ mouse, which has been shown to exhibit marked methacholine-induced AHR that is independent of allergen sensitisation and challenge [99]. Quantitative locus analysis of the AHR revealed that this naïve AHR is conferred by a major locus on $\mathrm{A} / \mathrm{J}$ chromosome 2 and is an interacting locus on chromosome 6 [100]. Interestingly, chromosome 2 also contains the mouse orthologue of ADAM33.

\section{CONCLUSIONS}

Airway remodelling is a potentially important consequence of asthma. Although there has been considerable effort to design in vitro, in vivo and in silico systems to determine the cells and molecules responsible for the changes in tissue pathology, it is clear that there are still many unanswered questions. Further effort is needed to determine the relationship between changes in pathology and physiology before treatment regimens to prevent or resolve established remodelling can be created. It is clear that an integrated approach is needed to coordinate laboratory models with clinical studies to determine some of the answers to the questions discussed previously.

\section{REFERENCES}

1 Vignola AM, Chanez $P$, Siena L, Chiappara G, Bonsignore G, Bousquet J. Airways remodelling in asthma. Pulm Pharmacol Ther 1998; 11: 359-367.

2 Stewart AG. Airway wall remodelling and hyperresponsiveness: modelling remodelling in vitro and in vivo. Pulm Pharmacol Ther 2001; 14: 255-265.

3 Gray TE, Guzman K, Davis CW, Abdullah LH, Nettesheim P. Mucociliary differentiation of serially passaged normal human tracheobronchial epithelial cells. Am J Respir Cell Mol Biol 1996; 14: 104-112.

4 Kikuchi T, Shively JD, Foley JS, Drazen JM, Tschumperlin DJ. Differentiation-dependent responsiveness of bronchial epithelial cells to IL-4/13 stimulation. Am J Physiol Lung Cell Mol Physiol 2004; 287: L119-L126.

5 Puddicombe SM, Torres-Lozano C, Richter A, et al. Increased expression of p21 ${ }^{\text {waf }}$ cyclin-dependent kinase inhibitor in asthmatic bronchial epithelium. Am J Respir Cell Mol Biol 2003; 28: 61-68.

6 Puddicombe SM, Polosa R, Richter A, et al. Involvement of the epidermal growth factor receptor in epithelial repair in asthma. FASEB J 2000; 14: 1362-1374.

7 Holgate ST, Lackie PM, Howarth $\mathrm{PH}$, et al. Invited lecture: activation of the epithelial mesenchymal trophic 
unit in the pathogenesis of asthma. Int Arch Allergy Immunol 2001; 124: 253-258.

8 Holgate ST, Davies DE, Rorke S, et al. ADAM 33 and its association with airway remodeling and hyperresponsiveness in asthma. Clin Rev Allergy Immunol 2004; 27: 23-34.

9 Kelly MM, Leigh R, Bonniaud $\mathrm{P}$, et al. Epithelial expression of profibrotic mediators in a model of allergen-induced airway remodeling. Am J Respir Cell Mol Biol 2005; 32: 99-107.

10 Brightling CE, Bradding P, Symon FA, Holgate ST, Wardlaw AJ, Pavord ID. Mast-cell infiltration of airway smooth muscle in asthma. N Engl J Med 2002; 346: 1699-1705.

11 Robinson DS. The role of the mast cell in asthma: induction of airway hyperresponsiveness by interaction with smooth muscle? J Allergy Clin Immunol 2004; 114: 58-65.

12 Macklem PT. A theoretical analysis of the effect of airway smooth muscle load on airway narrowing. Am J Respir Crit Care Med 1996; 153: 83-89.

13 Chesney J, Bucala R. Peripheral blood fibrocytes: novel fibroblast-like cells that present antigen and mediate tissue repair. Biochem Soc Trans 1997; 25: 520-524.

14 Schmidt M, Sun G, Stacey MA, Mori L, Mattoli S. Identification of circulating fibrocytes as precursors of bronchial myofibroblasts in asthma. J Immunol 2003; 171: 380-389.

15 Chakir J, Page N, Hamid Q, Laviolette M, Boulet LP, Rouabhia M. Bronchial mucosa produced by tissue engineering: a new tool to study cellular interactions in asthma. J Allergy Clin Immunol 2001; 107: 36-40.

16 Jaffar Z, Roberts K, Pandit A, Linsley P, Djukanovic R, Holgate S. B7 costimulation is required for IL-5 and IL-13 secretion by bronchial biopsy tissue of atopic asthmatic subjects in response to allergen stimulation. Am J Respir Cell Mol Biol 1999; 20: 153-162.

17 Morin C, Proteau S, Rousseau E, Brayden J. Organcultured airway explants: a new model of airway hyperresponsiveness. Exp Lung Res 2005; 31: 719-744.

18 Lloyd CM, Gonzalo JA, Coyle AJ, Gutierrez-Ramos JC. Mouse models of allergic airway disease. Adv Immunol 2001; 77: 263-295.

19 Kumar RK, Foster PS. Modeling allergic asthma in mice: pitfalls and opportunities. Am J Respir Cell Mol Biol 2002; 27: $267-272$.

20 Lloyd CM, Robinson DS, McMillan SJ. Modelling airway remodelling in asthma. Drug Discov Today: Disease Models 2004; 1: 425-430.

21 Johnson JR, Wiley RE, Fattouh R, et al. Continuous exposure to house dust mite elicits chronic airway inflammation and structural remodeling. Am J Respir Crit Care Med 2004; 169: 378-385.

22 Kumar RK, Herbert C, Yang M, Koskinen AM, McKenzie AN, Foster PS. Role of interleukin-13 in eosinophil accumulation and airway remodelling in a mouse model of chronic asthma. Clin Exp Allergy 2002; 32: 1104-1111.

23 Foster PS, Webb DC, Yang M, Herbert C, Kumar RK. Dissociation of $\mathrm{T}$ helper type 2 cytokine-dependent airway lesions from signal transducer and activator of transcription 6 signalling in experimental chronic asthma. Clin Exp Allergy 2003; 33: 688-695.
24 Yang G, Li L, Volk A, et al. Therapeutic dosing with antiinterleukin-13 monoclonal antibody inhibits asthma progression in mice. J Pharmacol Exp Ther 2005; 313: 8-15.

25 Cho JY, Miller M, Baek KJ, et al. Inhibition of airway remodeling in IL-5-deficient mice. J Clin Invest 2004; 113: 551-560.

26 Humbles AA, Lloyd CM, McMillan SJ, et al. A critical role for eosinophils in allergic airways remodeling. Science 2004; 305: 1776-1779.

27 Temann UA, Geba GP, Rankin JA, Flavell RA. Expression of interleukin 9 in the lungs of transgenic mice causes airway inflammation, mast cell hyperplasia, and bronchial hyperresponsiveness. J Exp Med 1998; 188: 1307-1320.

28 Zhu Z, Homer RJ, Wang Z, et al. Pulmonary expression of interleukin-13 causes inflammation, mucus hypersecretion, subepithelial fibrosis, physiologic abnormalities, and eotaxin production. J Clin Invest 1999; 103: 779-788.

29 Snibson KJ, Bischof RJ, Slocombe RF, Meeusen EN. Airway remodelling and inflammation in sheep lungs after chronic airway challenge with house dust mite. Clin Exp Allergy 2005; 35: 146-152.

30 Coffman RL, Hessel EM. Nonhuman primate models of asthma. J Exp Med 2005; 201: 1875-1879.

31 Hart TK, Cook RM, Zia-Amirhosseini P, et al. Preclinical efficacy and safety of mepolizumab (SB-240563), a humanized monoclonal antibody to IL-5, in cynomolgus monkeys. J Allergy Clin Immunol 2001; 108: 250-257.

32 Schelegle ES, Gershwin LJ, Miller LA, et al. Allergic asthma induced in rhesus monkeys by house dust mite (Dermatophagoides farinae). Am J Pathol 2001; 158: 333-341.

33 Fanucchi MV, Schelegle ES, Baker GL, et al. Immunostimulatory oligonucleotides attenuate airways remodeling in allergic monkeys. Am J Respir Crit Care Med 2004; 170: 1153-1157.

34 Tran MU, Weir AJ, Fanucchi MV, et al. Smooth muscle hypertrophy in distal airways of sensitized infant rhesus monkeys exposed to house dust mite allergen. Clin Exp Allergy 2004; 34: 1627-1633.

35 Bentley AM, Kay AB, Durham SR. Human late asthmatic reactions. Clin Exp Allergy 1997; 27: Suppl. 1, 71-86.

36 Phipps S, Benyahia F, Ou T-T, Barkans J, Robinson DS, Kay AB. Acute allergen-induced airway remodeling in atopic asthma. Am J Respir Cell Mol Biol 2004; 31: 626-632.

37 Wiggs BR, Moreno R, Hogg JC, Hilliam C, Pare PD. A model of the mechanics of airway narrowing. J Appl Physiol 1990; 69: 849-860.

38 Lambert RK, Wiggs BR, Kuwano K, Hogg JC, Pare PD. Functional significance of increased airway smooth muscle in asthma and COPD. J Appl Physiol 1993; 74: 2771-2781.

39 Seow CY, Wang L, Pare PD. Airway narrowing and internal structural constraints. J Appl Physiol 2000; 88: $527-533$

40 Wiggs BR, Hrousis CA, Drazen JM, Kamm RD. On the mechanism of mucosal folding in normal and asthmatic airways. J Appl Physiol 1997; 83: 1814-1821.

41 Kitaoka H, Takaki R, Suki B. A three-dimensional model of the human airway tree. J Appl Physiol 1999; 87: 2207-2217.

42 Martonen T, Fleming J, Schroeter J, Conway J, Hwang D. In silico modeling of asthma. Adv Drug Deliv Rev 2003; 55: 829-849. 
43 Bai TR, Knight DA. Structural changes in the airways in asthma: observations and consequences. Clin Sci (Lond) 2005; 108: 463-477.

44 Wardlaw AJ, Dunnette S, Gleich GJ, Collins JV, Kay AB. Eosinophils and mast cells in bronchoalveolar lavage in subjects with mild asthma. Relationship to bronchial hyperreactivity. Am Rev Respir Dis 1988; 137: 62-69.

45 Bousquet J, Chanez P, Lacoste JY, et al. Eosinophilic inflammation in asthma. N Engl J Med 1990; 323: 1033-1039.

46 Brusasco V, Crimi E, Gianiorio P, Lantero S, Rossi GA. Allergen-induced increase in airway responsiveness and inflammation in mild asthma. J Appl Physiol 1990; 69: 2209-2214

47 Lotvall J, Inman M, O'Byrne P. Measurement of airway hyperresponsiveness: new considerations. Thorax 1998; 53: 419-424.

48 Djukanovic R, Wilson JW, Britten KM, et al. Effect of an inhaled corticosteroid on airway inflammation and symptoms in asthma. Am Rev Respir Dis 1992; 145: 669-674.

49 Sont JK, Willems LN, Bel EH, Van Krieken JH, Vandenbroucke JP, Sterk PJ. Clinical control and histopathologic outcome of asthma when using airway hyperresponsiveness as an additional guide to long-term treatment. The AMPUL Study Group. Am J Respir Crit Care Med 1999; 159: 1043-1051.

50 Boulet LP, Turcotte H, Laviolette M, et al. Airway hyperresponsiveness, inflammation, and subepithelial collagen deposition in recently diagnosed versus longstanding mild asthma. Influence of inhaled corticosteroids. Am J Respir Crit Care Med 2000; 162: 1308-1313.

51 Lange P, Parner J, Vestbo J, Schnohr P, Jensen G. A 15year follow-up study of ventilatory function in adults with asthma. N Engl J Med 1998; 339: 1194-1200.

52 Ulrik CS, Backer V. Nonreversible airflow obstruction in life-long nonsmokers with moderate to severe asthma. Eur Respir J 1999; 14: 892-896.

53 Flood-Page P, Menzies-Gow A, Phipps S, et al. Anti-IL-5 treatment reduces deposition of ECM proteins in the bronchial subepithelial basement membrane of mild atopic asthmatics. J Clin Invest 2003; 112: 1029-1036.

54 Nakano Y, Muller NL, King GG, et al. Quantitative assessment of airway remodeling using high-resolution CT. Chest 2002; 122: 271S-275S.

55 Nakano Y, Wong JC, de Jong PA, et al. The prediction of small airway dimensions using computed tomography. Am J Respir Crit Care Med 2005; 171: 142-146.

56 Kasahara K, Shiba K, Ozawa T, Okuda K, Adachi M. Correlation between the bronchial subepithelial layer and whole airway wall thickness in patients with asthma. Thorax 2002; 57: 242-246.

57 Pare PD. Airway hyperresponsiveness in asthma: geometry is not everything!. Am J Respir Crit Care Med 2003; 168: 913-914.

58 Niimi A, Matsumoto H, Takemura M, Ueda T, Chin K, Mishima M. Relationship of airway wall thickness to airway sensitivity and airway reactivity in asthma. Am J Respir Crit Care Med 2003; 168: 983-988.

59 Halayko AJ, Solway J. Molecular mechanisms of phenotypic plasticity in smooth muscle cells. J Appl Physiol 2001; 90: 358-368.
60 Henderson WR Jr, Lewis DD, Albert RK, et al. The importance of leukotrienes in airway inflammation in a mouse model of asthma. J Exp Med 1996; 184: 1483-1494.

61 Tanaka H, Masuda T, Tokuoka S, et al. The effect of allergen-induced airway inflammation on airway remodeling in a murine model of allergic asthma. Inflamm Res 2001; 50: 616-624.

62 Leigh R, Ellis R, Wattie J, et al. Dysfunction and remodeling of the mouse airway persist after resolution of acute allergen-induced airway inflammation. Am J Respir Cell Mol Biol 2002; 27: 526-535.

63 Johnson JR, Wiley RE, Fattouh R, et al. Continuous exposure to house dust mite elicits chronic airway inflammation and structural remodeling. Am J Respir Crit Care Med 2004; 169: 378-385.

64 Leigh R, Ellis R, Wattie JN, et al. Type 2 cytokines in the pathogenesis of sustained airway dysfunction and airway remodeling in mice. Am J Respir Crit Care Med 2004; 169: 860-867.

65 Masuda T, Tanaka H, Komai M, et al. Mast cells play a partial role in allergen-induced subepithelial fibrosis in a murine model of allergic asthma. Clin Exp Allergy 2003; 33: 705-713

66 McMillan SJ, Xanthou G, Lloyd CM. manipulation of allergen-induced airway remodeling by treatment with anti-TGF- $\beta$ antibody: effect on the Smad signaling pathway. J Immunol 2005; 174: 5774-5780.

67 Green RH, Brightling CE, Woltmann G, Parker D, Wardlaw AJ, Pavord ID. Analysis of induced sputum in adults with asthma: identification of subgroup with isolated sputum neutrophilia and poor response to inhaled corticosteroids. Thorax 2002; 57: 875-879.

68 Holgate ST, Davies DE, Lackie PM, Wilson SJ, Puddicombe SM, Lordan JL. Epithelial-mesenchymal interactions in the pathogenesis of asthma. J Allergy Clin Immunol 2000; 105: 193-204.

69 Baldwin L, Roche WR. Does remodelling of the airway wall precede asthma? Paediatr Respir Rev 2002; 3: 315-320.

70 Cutz E, Levison H, Cooper DM. Ultrastructure of airways in children with asthma. Histopathology 1978; 2: 407-421.

71 Pohunek P, Warner JO, Turzikova J, Kudrmann J, Roche WR. Markers of eosinophilic inflammation and tissue re-modelling in children before clinically diagnosed bronchial asthma. Pediatr Allergy Immunol 2005; 16: 43-51.

72 Martinez FD, Wright AL, Taussig LM, Holberg CJ, Halonen M, Morgan WJ. Asthma and wheezing in the first six years of life. The Group Health Medical Associates. N Engl J Med 1995; 332: 133-138.

73 Merkus PJ, Essen-Zandvliet EE, Kouwenberg JM, et al. Large lungs after childhood asthma. A case-control study. Am Rev Respir Dis 1993; 148: 1484-1489.

74 Payne DNR, Rogers AV, Adelroth E, et al. Early thickening of the reticular basement membrane in children with difficult asthma. Am J Respir Crit Care Med 2003; 167: 78-82.

75 Hogg JC, Williams J, Richardson JB, Macklem PT, Thurlbeck WM. Age as a factor in the distribution of lower-airway conductance and in the pathologic anatomy of obstructive lung disease. N Engl J Med 1970; 282: 1283-1287. 
76 Bai TR. Abnormalities in airway smooth muscle in fatal asthma. Am Rev Respir Dis 1990; 141: 552-557.

77 Saglani S, Malmstrom K, Pelkonen AS, et al. Airway remodeling and inflammation in symptomatic infants with reversible airflow obstruction. Am J Respir Crit Care Med 2005; 171: 722-727.

78 Chetta A, Foresi A, Del Donno M, Bertorelli G, Pesci A, Olivieri D. Airways remodeling is a distinctive feature of asthma and is related to severity of disease. Chest 1997; 111: 852-857.

79 van Velzen E, van den Bos JW, Benckhuijsen JA, van Essel T, de Bruijn R, Aalbers R. Effect of allergen avoidance at high altitude on direct and indirect bronchial hyperresponsiveness and markers of inflammation in children with allergic asthma. Thorax 1996; 51: 582-584.

80 Saetta M, Maestrelli P, Turato G, et al. Airway wall remodeling after cessation of exposure to isocyanates in sensitized asthmatic subjects. Am J Respir Crit Care Med 1995; 151: 489-494.

81 Kumar RK, Herbert C, Webb DC, Li L, Foster PS. Effects of anticytokine therapy in a mouse model of chronic asthma. Am J Respir Crit Care Med 2004; 170: 1043-1048.

82 McMillan S, Lloyd C. Prolonged allergen challenge in mice leads to persistent airway remodelling. Clin Exp Allergy 2004; 34: 497-507.

83 Ward C, Pais M, Bish R, et al. Airway inflammation, basement membrane thickening and bronchial hyperresponsiveness in asthma. Thorax 2002; 57: 309-316.

84 Chetta A, Zanini A, Foresi A, et al. Vascular component of airway remodeling in asthma is reduced by high dose of fluticasone. Am J Respir Crit Care Med 2003; 167: 751-757.

85 Jeffery PK, Godfrey RW, Adelroth E, Nelson F, Rogers A, Johansson S-A. Effects of treatment on airway inflammation and thickening of basement membrane reticular collagen in asthma. A quantitive light and electron microscopic study. Am Rev Respir Dis 1992; 145: 890-899.

86 Boulet LP. Comparative improvement of asthma symptoms and expiratory flows after corticosteroid treatment: a method to assess the effect of corticosteroids on large versus small airways? Respir Med 2006; 100: 496-502.

87 Ward C, Walters H. Airway wall remodelling: the influence of corticosteroids. Curr Opin Allergy Clin Immunol 2005; 5: 43-48.
88 Gerhardt SG, McDyer JF, Girgis RE, Conte JV, Yang SC, Orens JB. Maintenance azithromycin therapy for bronchiolitis obliterans syndrome: results of a pilot study. Am J Respir Crit Care Med 2003; 168: 121-125.

89 Christie PE, Jonas M, Tsai CH, Chi EY, Henderson WR Jr. Increase in laminin expression in allergic airway remodelling and decrease by dexamethasone. Eur Respir J 2004; 24: 107-115.

90 McMillan SJ, Xanthou G, Lloyd CM. Therapeutic administration of Budesonide ameliorates allergen-induced airway remodelling. Clin Exp Allergy 2005; 35: 388-396.

91 Henderson WR Jr, Tang LO, Chu SJ, et al. A role for cysteinyl leukotrienes in airway remodeling in a mouse asthma model. Am J Respir Crit Care Med 2002; 165: 108-116.

92 Halapi E, Hakonarson H. Recent development in genomic and proteomic research for asthma. Curr Opin Pulm Med 2004; 10: 22-30.

93 Postma DS, Bleecker ER, Amelung PJ, et al. Genetic susceptibility to asthma - bronchial hyperresponsiveness coinherited with a major gene for atopy. $N$ Engl J Med 1995; 333: 894-900.

94 Van Eerdewegh P, Little RD, Dupuis J, et al. Association of the ADAM33 gene with asthma and bronchial hyperresponsiveness. Nature 2002; 418: 426-430.

95 Simpson A, Maniatis N, Jury F, et al. Polymorphisms in A disintegrin and metalloprotease 33 (ADAM33) predict impaired early-life lung function. Am J Respir Crit Care Med 2005; 172: 55-60.

96 van Diemen CC, Postma DS, Vonk JM, Bruinenberg M, Schouten JP, Boezen HM. A disintegrin and metalloprotease 33 polymorphisms and lung function decline in the general population. Am J Respir Crit Care Med 2005; 172: 329-333.

97 Drazen JM, Arm JP, Austen KF. Sorting out the cytokines of asthma. J Exp Med 1996; 183: 1-5.

98 Shinagawa K, Kojima M. Mouse model of airway remodeling: strain differences. Am J Respir Crit Care Med 2003; 168: 959-967.

99 De Sanctis GT, Merchant M, Beier DR, et al. Quantitative locus analysis of airway hyperresponsiveness in $\mathrm{A} / \mathrm{J}$ and C57BL/6J mice. Nat Genet 1995; 11: 150-154.

100 Ackerman KG, Huang H, Grasemann H, et al. Interacting genetic loci cause airway hyperresponsiveness. Physiol Genomics 2005; 21: 105-111. 